\title{
Stat5a serine 725 and 779 phosphorylation is a prerequisite for hematopoietic transformation
}

\author{
Katrin Friedbichler, ${ }^{1}$ Marc A. Kerenyi, ${ }^{2}$ Boris Kovacic, ${ }^{3}$ Geqiang Li, ${ }^{4}$ Andrea Hoelbl, ${ }^{5}$ Saliha Yahiaoui, ${ }^{6}$ Veronika Sexl, ${ }^{5}$ \\ Ernst W. Müllner, ${ }^{7}$ Sabine Fajmann, ${ }^{5}$ Sabine Cerny-Reiterer, ${ }^{8}$ Peter Valent, $, 8,9$ Hartmut Beug, ${ }^{3}$ Fabrice Gouilleux, ${ }^{10}$ \\ Kevin D. Bunting, ${ }^{4}$ and Richard Morigg ${ }^{1}$ \\ ${ }^{1}$ Ludwig Boltzmann Institute for Cancer Research (LBI-CR), Vienna, Austria; '2Department of Hematology/Oncology, Children's Hospital, Harvard Medical \\ School, Boston, MA; ${ }^{3}$ Research Institute of Molecular Pathology (IMP), Vienna, Austria; ${ }^{4}$ Division of Hematology/Oncology, Department of Medicine, School of \\ Medicine, Case Western Reserve University, Cleveland, $\mathrm{OH} ;{ }^{5}$ Institute of Pharmacology, Medical University of Vienna, Vienna, Austria; ${ }^{6}$ Inserm U925, Faculté \\ de Médecine, Université de Picardie J. Verne, Amiens, France; ${ }^{7}$ Max F. Perutz Laboratories, Department of Medical Biochemistry, and ${ }^{8}$ Department of Internal \\ Medicine I, Division of Hematology and Hemostaseology, Medical University of Vienna, Vienna, Austria; ' 2 udwig Boltzmann Cluster Oncology, Vienna, Austria; \\ and ${ }^{10}$ Centre National de la Recherche Scientifique (CNRS) UMR 6239, Université F. Rabelais, UFR Médecine, Tours, France
}

\begin{abstract}
Stat5 transcription factors are essential gene regulators promoting proliferation, survival, and differentiation of all hematopoietic cell types. Mutations or fusions of oncogenic tyrosine kinases often result in constitutive Stat 5 activation. We have modeled persistent Stat 5 activity by using an oncogenic Stat5a variant (cS5). To analyze the hitherto unrecognized role of Stat5 serine phosphorylation in this context, we have generated cS5 constructs with mutated C-terminal serines 725 and
\end{abstract}

779 , either alone or in combination. Genetic complementation assays in primary Stat $5^{\text {null/null }}$ mast cells and Stat $5^{\Delta \mathrm{N}} \mathrm{T}$ cells demonstrated reconstitution of proliferation with these mutants. Similarly, an in vivo reconstitution experiment of transduced Stat $5^{\text {null/null }}$ fetal liver cells transplanted into irradiated wild-type recipients revealed that these mutants exhibit biologic activity in lineage differentiation. By contrast, the leukemogenic potential of cS5 in bone marrow transplants de- creased dramatically in cS5 single-serine mutants or was completely absent upon loss of both serine phosphorylation sites. Our data suggest that Stat5a serine phosphorylation is a prerequisite for cS5mediated leukemogenesis. Hence, interference with Stat5a serine phosphorylation might provide a new therapeutic option for leukemia and myeloid dysplasias without affecting major functions of Stat5 in normal hematopoiesis. (Blood. 2010;116(9): 1548-1558)

\section{Introduction}

Hematopoietic development is regulated by cytokine- or growth factor-activated signaling pathways, among which the Janus kinase (Jak)/signal transducer and activator of transcription (Stat) pathway plays a major role. ${ }^{1-3}$ Four Jak kinases and 7 Stat proteins regulate a wide spectrum of cellular functions such as proliferation, survival, and differentiation. ${ }^{4}$ Stats are latent transcription factors that constantly shuttle between the nucleus and the cytoplasm. Their activity is tightly regulated by protein tyrosine kinases. Phosphorylation of a positionally conserved tyrosine residue triggers dimerization, efficient nuclear translocation, and subsequent binding of Stats to specific promoter sequences. ${ }^{5}$ Target genes regulated by Stat 5 proteins and their cofactors are essential for hematopoietic stem cell maintenance, lineage commitment, self-renewal, and survival of committed hematopoietic progenitors as well as for mature cells of both myeloerythroid and lymphoid lineage. ${ }^{6-8}$ Although there is considerable functional overlap, Stat5a and Stat5b proteins also have distinct functions due to isoformspecific differences in mRNA levels, ${ }^{3,5}$ nucleocytoplasmic shuttling, 9,10 and activation by tyrosine and/or serine phosphorylation. ${ }^{11}$

Hyperactivated Stat5a and Stat5b proteins have been implicated in several hematopoietic malignancies and many solid tumors. ${ }^{1-3}$ Yet to date, there are no reports of activating mutations in Stat5 proteins. Persistent tyrosine phosphorylation of Stat5 was found to be caused by deregulated cytokine signaling ${ }^{12}$ or perturbation of upstream molecules, which mostly results from receptor mutations or chromosomal rearrangement. Well-known examples of this are the point mutation Jak2-V617F ${ }^{13,14}$ and chromosomal translocations such as Bcr-Abl. ${ }^{15,16}$ Furthermore, it has been shown that the presence of Stat5 proteins is required for Bcr-Abl-induced transformation and development of leukemia. ${ }^{17}$ This is why Stat5 proteins and their activation status are thought to have a key role in leukemogenesis. Moreover, the transforming capacity of oncogenic Stat 5 is not limited to its role as a transcription factor in the nucleus. Recent studies have demonstrated that oncogenic Stat5a also has a cytoplasmic role that links Jak/Stat signaling to the activation of the PI3K-Akt-mTOR signaling pathway via Gab2 $2^{18-20}$ and that Stat5 acts as a repressor of $\mathrm{Bcl}-2$ member-regulating microRNAs (miRNAs) $15 / 16 .^{21}$

In addition to tyrosine phosphorylation, the activity of several Stat proteins was shown to be modulated by serine phosphorylation (reviewed in Decker and Kovarik ${ }^{22}$ ). In the highly homologous Stat5a and Stat5b proteins, distinct serine residues in the carboxyterminal transactivation domain, the least conserved region of Stat proteins, ${ }^{2,3}$ were found to be phosphorylated. These serine moieties, however, are located in perfectly conserved Pro-Ser-Pro (PSP) motifs at positions 725 (Stat5a) and 730 (Stat5b). ${ }^{11}$ In
Submitted December 14, 2009; accepted May 15, 2010. Prepublished online as Blood First Edition paper, May 27, 2010; DOI 10.1182/blood-2009-12-258913.
The publication costs of this article were defrayed in part by page charge payment. Therefore, and solely to indicate this fact, this article is hereby marked "advertisement" in accordance with 18 USC section 1734. 
addition, Stat5a harbors a unique serine residue within a Leu-SerPro (LSP) motif (Ser779), ${ }^{23}$ which is also found in mouse, rat, pig, and human Stat5a sequences. As shown by Beuvink et al, Ser779 is the major site of serine phosphorylation in Stat5a. ${ }^{23}$ However, the biologic significance of Stat5 serine phosphorylation and particularly its proto-oncogenic functions have not yet been clarified. Although serine phosphorylation has been demonstrated to substantially modulate the transcriptional activity of Stat1 and Stat3 proteins, ${ }^{22}$ it was long thought to have a minor role in Stat5 function. ${ }^{11,23-27}$ Using a mouse transplantation model, ${ }^{28,29}$ the present study is the first to provide evidence that serine phosphorylation plays a crucial role in Stat5-driven leukemogenesis.

\section{Methods}

\section{Human patient samples}

Primary leukemic cells were isolated from bone marrow (BM) aspirates or peripheral blood (PB) using Ficoll and washed in ice-cold phosphatebuffered saline (PBS) supplemented with complete protease inhibitors (Roche) and phosphatase blockers $\left(1 \mathrm{mM} \mathrm{NaF}, 1 \mathrm{mM} \mathrm{Na} \mathrm{VO}_{4}, 10 \mathrm{mM}\right.$ $\beta$-glycerophosphate). Informed consent was obtained in each case before PB donation or BM puncture. Characteristics of the patients are shown in supplemental Table 1 (available on the Blood Web site; see the Supplemental Materials link at the top of the online article). For control purposes, 2 patients with normal BM (complete remission from acute myeloid leukemia [AML] after chemotherapy) were examined. The study was approved by the Institutional Review Board of the Medical University of Vienna and was conducted in accordance with the Declaration of Helsinki.

\section{Animals, primary cell isolation, retroviral infection, proliferation assays, and transplantation}

BM transplantation. BM was harvested from femurs and tibias of 6-week-old male mice (C57/B6xSv129j F1) and cultured for 24 hours in Dulbecco modified Eagle medium (DMEM), 15\% fetal calf serum (FCS) containing interleukin-3 (IL-3, 25 ng/mL; R\&D Systems), IL-6 (50 ng/mL; R\&D Systems), and stem cell factor (SCF, $200 \mathrm{ng} / \mathrm{mL}$; stem cell medium [SCM]). In the presence of $6 \mu \mathrm{g} / \mathrm{mL}$ polybrene, cells were subsequently cocultured with confluent ecotropic producer cell lines for 72 hours in SCM. After 48 hours of coculture, BM cells were analyzed for green fluorescent protein (GFP) expression by flow cytometry. Lethally irradiated (10 Gy) wild-type (WT) female recipients (C57/B6xSv129j F1) were reconstituted with an equal amount of transduced $\left(\mathrm{GFP}^{+}\right)$LSK $\left(\right.$ Lineage $\left.{ }^{-} \mathrm{Sca}^{+} \mathrm{c}-\mathrm{Kit}^{+}\right)$cells by tail vein injection $\left(6 \times 10^{3}\right)$. Transplanted mice were monitored for disease onset by blood analysis at regular intervals, starting 4 weeks after transplantation. All transplanted mice were housed in a specific pathogen-free environment and monitored daily. The study was performed under the animal license form BMWF-66.009/0139-C/ GT/2007 according to standard ethical guidelines.

Fetal liver cell transplantation. Stat5ab $\mathrm{b}^{\text {null/null }}$ fetal liver cells (CD45.2) were transduced with either the vector control or WT Stat5a or the S5-SASA variant. All 3 groups of cells were transplanted into lethally irradiated CD45.1 recipient mice. Two months after transplantation, the mice were bled and analyzed for donor engraftment, GFP expression, and lineage repopulation (CD8, B220, Gr-1, and Ter-119). Donor cells were identified, and the absolute number of donor-derived cells of the various lineages quantified by CD45.2 staining. ${ }^{30}$ GFP ${ }^{\text {tg }}$ mice served as positive controls.

T-cell complementation assay. T cells were isolated from the spleens of 8-week-old WT and Stat5a/b-deficient (Stat $5^{\Delta \mathrm{N} / \Delta \mathrm{N}}$ ) mice without splenomegaly. ${ }^{31,32}$ Naive splenic T cells were stimulated with a monoclonal $\alpha$-CD3 antibody (145.2C11, 1:1000; Pharmingen) and IL-2 (500 U/mL; R\&D Systems). Freshly isolated primary WT as well as Stat5-deficient splenocytes were cocultured with confluent ecotropic producer cell lines for 72 hours, in the presence of $500 \mathrm{U} / \mathrm{mL} \mathrm{IL-2}$ and $6 \mu \mathrm{g} / \mathrm{mL}$ polybrene. Then the cells were expanded in the presence of $500 \mathrm{U} / \mathrm{mL} \mathrm{IL}-2$ for 14 days to enrich for transduced cells. Having reached an infection rate of $40 \%$ to $80 \%$, proliferating T cells were deprived of IL-2 for 18 hours and subsequently incubated as triplicates in round-bottom 96-well plates for 36 hours at a density of $1 \times 10^{5}$ cells per well in medium supplemented with different concentrations of IL-2. In the last 18 hours, ${ }^{3} \mathrm{H}$-thymidine $(0.2 \mu \mathrm{Ci} /$ well $)$ as well as a repeat dose of IL- 2 were added. The proliferation rate was determined by measuring thymidine incorporation with a $\beta$-counter.

\section{Mouse peripheral blood hematology and fluorescence-activated cell sorting}

PB was obtained by puncturing the retro-orbital venous sinus with a microcapillary tube. To obtain white blood cell (WBC) counts, PB was analyzed using the Vet animal blood counter (Scil animal care). For multilineage analysis, PB cells were stained with fluorescence-conjugated antibodies (all from BD Biosciences) against Ter-119 (phycoerythrin [PE]), GR-1 (allophycocyanin [APC]), Mac-1 (PE-Cy7), CD19 (APC-Cy7), CD3 (PerCp), Sca-1 (PE-Cy7), c-Kit (Cychrome), Flt3 (PE), and Thy1 (APC). An annexin V (PE; BD Biosciences) antibody was used to assess apoptosis in transduced $\mathrm{Ba} / \mathrm{F} 3$ cells. The samples were analyzed by flow cytometry (FACSCanto; Becton Dickinson).

\section{Cell culture, plasmids, transfection of $\mathrm{Ba} / \mathrm{F} 3$ cells, and reagents}

All Stat5a mutants were generated by polymerase chain reaction (PCR) mutagenesis and verified by sequencing. A constitutively active mutant of Stat5a (cS5, also known as $\mathrm{S} 11 \mathrm{~F}^{33}$ which in murine Stat5a is in fact a mutation of serine 710 to phenylalanine) was used as described previously. ${ }^{28,29}$ Stat5a serine mutants were generated by substituting alanines for serines at amino acid positions 725 and/or $779 .{ }^{23}$ All constructs were cloned into the murine bicistronic retroviral vector pMSCV-IRES-eGFP. NIH-3T3based, ecotropic, replication-incompetent, retroviral gp + E86 producer cell lines were generated and selected for high viral titers $\left(\sim 5 \times 10^{6}\right.$ particles/ $\mathrm{mL}$ as tested in NIH-3T3 cells) by FACS sorting. Producer cells were maintained in DMEM, whereas Ba/F3, K562, Ku-812, Mv4.11, and MOLM13 cells were grown in RPMI 1640. Both media were supplemented with $10 \%$ FCS, L-glutamine $(2 \mathrm{mM})$, and penicillin/streptomycin $(10 \mathrm{U} / \mathrm{mL}$, $10 \mu \mathrm{g} / \mathrm{mL}$ ). Parental Ba/F3 cells were grown in the presence of IL-3 $(2 \mathrm{ng} / \mathrm{mL})$.

\section{Immunohistochemistry and immunocytochemistry}

Immunohistochemistry was performed on sections prepared from paraffinembedded formalin-fixed spleen, liver, and BM specimens using hematoxylin and eosin (H\&E) staining. Blood smears and cytospins were stained with the Hemacolor rapid staining kit (Merck). Cytospins were prepared at a maximal density of $2 \times 10^{5}$ cells $/ \mathrm{mL}$. Stained sections and cytospins were analyzed by light microscopy. Images were captured with a PixeLINK camera and the corresponding acquisition software on a Zeiss Imager Z.1 (magnification $\times 100, \times 200$, or $\times 400$ ) at a color temperature of $1300 \mathrm{~K}$.

\section{Immunoblotting and DNA binding assays}

Sample preparation and Western blotting were performed using standard techniques. Hybond nitrocellulose membranes were incubated with antibodies raised against the following proteins: phosphotyrosine-Stat5ab (no. 05-495, Upstate; no. 71-6900, Zymed; no. 9351, Cell Signaling), phosphoserine-726/731-Stat5ab (no. 36153, Abcam), Stat5ab (no. 610192, BD Transduction Laboratories; N20, no. 836, C17, no. 835, Santa Cruz Biotechnology), HSC70 (no. 7298, loading control; Santa Cruz Biotechnology), tubulin (no. T-9026, loading control; Sigma-Aldrich), and actin (no. A5316, loading control; Sigma-Aldrich). Polyclonal rabbit sera against phosphoserines 725 and 779 of Stat5a (Stat5a-S725, H2N-DQAPS [PO3H2]PAVC-CONH2; Stat5a-S779, H2N-LDARLS[PO3H2]PPAGLFC $-\mathrm{CONH} 2$; Eurogentech) were generated and tested by the group of V.S. Eurogentech antibodies were used unless indicated otherwise. Electrophoretic mobility shift assay (EMSA) was performed for Stat5a dimers or tetramers using the $\beta$-casein response element alone or in tandem as 
described previously. ${ }^{2,28}$ For Stat5 supershifts, the C17 antibody (no. 835; Santa Cruz Biotechnology) was used.

\section{Statistics}

All values are represented as means plus or minus SD if not indicated otherwise. $\mathrm{WBC}$ and $\mathrm{Ba} / \mathrm{F} 3$ proliferation quantifications were evaluated for significance using the 2-tailed $t$ test. A $P$ value below .05 was considered significant for all analyses. Kaplan-Meier plots were analyzed for significance using the log-rank test. All calculations were performed using the GraphPad Prism 4 software.

\section{Results}

\section{Stat5a Ser725 and 779 are phosphorylated in human leukemic} cell lines and primary patient samples

The association of persistent Stat5 tyrosine phosphorylation with various hematologic malignancies is well established. However, there is still little information on the significance of serine phosphorylation for the proto-oncogenic functions of Stat5. We, therefore, investigated whether Stat5 is at all serine phosphorylated in leukemic cell lines expressing highly activated Stat5 as well as in primary patient samples. Specific antisera were generated that are directed against the mapped serine phosphorylation sites 725 and 779 of Stat5a, flanking the C-terminal transactivation domain. ${ }^{34,35}$ Two individually derived human chronic myeloid leukemia (CML) cell lines (K562 and Ku812) expressing Bcr-Abl p210 fusion kinase and 2 human AML cell lines (Mv4.11 and MOLM13) expressing Flt3-ITD and mixed-lineage leukemia (MLL)-fusion proteins were analyzed. As shown in Figure $1 \mathrm{~A}$, both bcr/abl ${ }^{+}$ CML lines displayed very high levels of Stat5 tyrosine phosphorylation (pY-Stat5), whereas the 2 AML cell lines exhibited lower levels. MOLM13 showed a very weak tyrosine phosphorylation signal, which may be attributed to reduced expression of total Stat5 protein. Similarly, Stat5a Ser779 phosphorylation, which was pronounced in the CML samples, was hardly detectable in the AML cell lines. Contrary, equal levels of Ser725 phosphorylation were found in all samples tested.

Furthermore, different $\mathrm{B}$ cell $(\mathrm{REH})$ and T-lineage acute lymphoblastic leukemia (T-ALL; CCRF-CEM, Molt-4, Jurkat, HPB-ALL), cutaneous T-cell lymphoma (CTCL; Mac-2A) as well as Burkitt lymphoma (Daudi, Ramos) and non-Burkitt lymphoma (RL-7) cell lines were analyzed for serine phosphorylation. All B-cell as well as the Mac-2A and the CCRF-CEM lines were found to express significant levels of Ser779 phosphorylation (Figure 1B). Ser725 phosphorylation was present to a lesser extent in all Tand B-cell lines except for Jurkat cells. A murine T-cell lymphoma line (EL-4), which was also included in the analysis, was positive for Ser725 and Ser779 phosphorylation. Interestingly, some cell lines displayed serine phosphorylation in the absence of significant pY-Stat5. While both Ser725/779 and tyrosine were phosphorylated in Mac-2A, Daudi, and to a lesser extent in Ramos and REH cells, there was marked serine phosphorylation in the transformed cell lines CCRF-CEM and RL-7 without pY-Stat5. It should be noted that the CML line K562 was used as a positive control, and the samples were transferred to the membranes by semidry instead of wet blotting in this assay only. However, it was confirmed by a separate experiment that the K562 signals in the Western blots displayed in Figure 1A and B were consistent (data not shown).

Moreover, primary samples from AML, ALL, and CML patients were subjected to Western blot analysis and were found to be strongly phosphorylated not only at the critical tyrosine 694 residue but also at serines 725 and 779 , which is unique to Stat5a. A comparison of leukemia patient samples and healthy individuals revealed that Stat5a Ser779 phosphorylation was not detected in healthy individuals, whereas serine 725 was found to be phosphorylated in all primary samples (Figure 1C).

\section{Fusion proteins regulating Stat5 activity induce Ser725/779 phosphorylation in $\mathrm{Ba} / \mathrm{F} 3$ cells}

To substantiate our findings, the well-established hematopoietic $\mathrm{Ba} / \mathrm{F} 3$ cell line was used. We generated several $\mathrm{Ba} / \mathrm{F} 3$ derivatives expressing various oncogenic mutations known to activate Stat5, including Bcr-Abl p210, Tel-Jak1/2/3, Tel-Abl, and oncogenic Stat5a (cS5) itself. Expression of these oncogenes rendered the cells capable of growing in the absence of IL-3. Starved and stimulated parental Ba/F3 cells as well as Stat5a (S5) overexpressing gpE +86 fibroblasts served as controls. As expected, parental $\mathrm{Ba} / \mathrm{F} 3$ cells, which had been starved for 20 hours, did not display pY-Stat5 phosphorylation. Under these conditions, Stat5 phosphorylation at the serine sites investigated was insignificant. By contrast, IL-3 stimulation resulted in highly tyrosine-phosphorylated Stat5 with concomitant phosphorylation of Ser725 and Ser779. Similar results were obtained in all $\mathrm{Ba} / \mathrm{F} 3$ cell lines harboring upstream fusion tyrosine kinases. Upon expression of these kinases, strong induction of Stat5a Tyr694, Ser725, and Ser779 phosphorylation was observed (Figure 1D). Overall, these data suggest that persistent Stat5 activation is associated with Stat5a Ser725/779 phosphorylation.

\section{Lack of serine phosphorylation does not alter the biochemical properties of Stat5a}

Different mutant Stat5a variants mimicking persistent Stat5 activation were used to study the role of serine phosphorylation in steady-state hematopoiesis and in the context of Stat 5 hyperactivation-driven leukemia. These constitutively active (cS5) and WT (S5) Stat5a constructs harbored point mutations at 2 distinct C-terminal serine residues (Ser725 and Ser779; Figure 2A). All mutants were subsequently expressed by pMSCV-IRES-eGFP retroviruses and packaging cell lines $(\mathrm{gpE}+86)$ were generated, which expressed comparably high titers of the respective retroviral particles. In gpE +86 cells, expression of serine phosphorylation was confirmed at residues Ser725 and Ser779, respectively (data not shown). The biochemical properties of all Stat5a variants were assessed to verify their activity. First, activating tyrosine phosphorylation (Y694) of the Stat5a variants was examined in stimulated and unstimulated 293T cells cotransfected with the erythropoietin (Epo) receptor (Figure 2B). Upon Epo stimulation, activated Stat5 was detected in S5 as well as cS5 constructs. In addition, all cS5 proteins showed persistent tyrosine phosphorylation without cytokine stimulation. It should be noted that Stat5a is constitutively phosphorylated on both serine residues when overexpressed in 293T cells (supplemental Figure 1). Furthermore, significant differences in DNA binding properties were excluded by EMSA using the $\beta$-casein response element (Figure 2C). The cS5 mutants were found to bind DNA even without cytokine stimulation and in the absence of serine phosphorylation. In a next step, $2 \times$ Stat5 responsive elements were used to test the tetramer formation capability of cS5 mutants, which is essential for their leukemogenic potential $^{28}$ (Figure 2D). No significant impairment of tetramer formation was observed in the cS5 variants, which all showed enhanced tetramerization on the $2 \times \beta$-casein element compared with WT Stat5a. Interestingly, mutation of either serine 725 or 779 

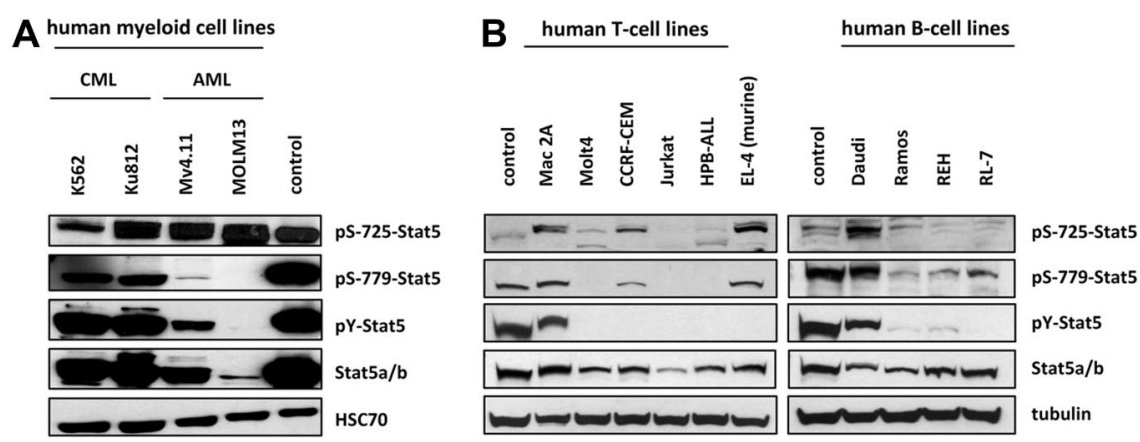

C primary patient samples

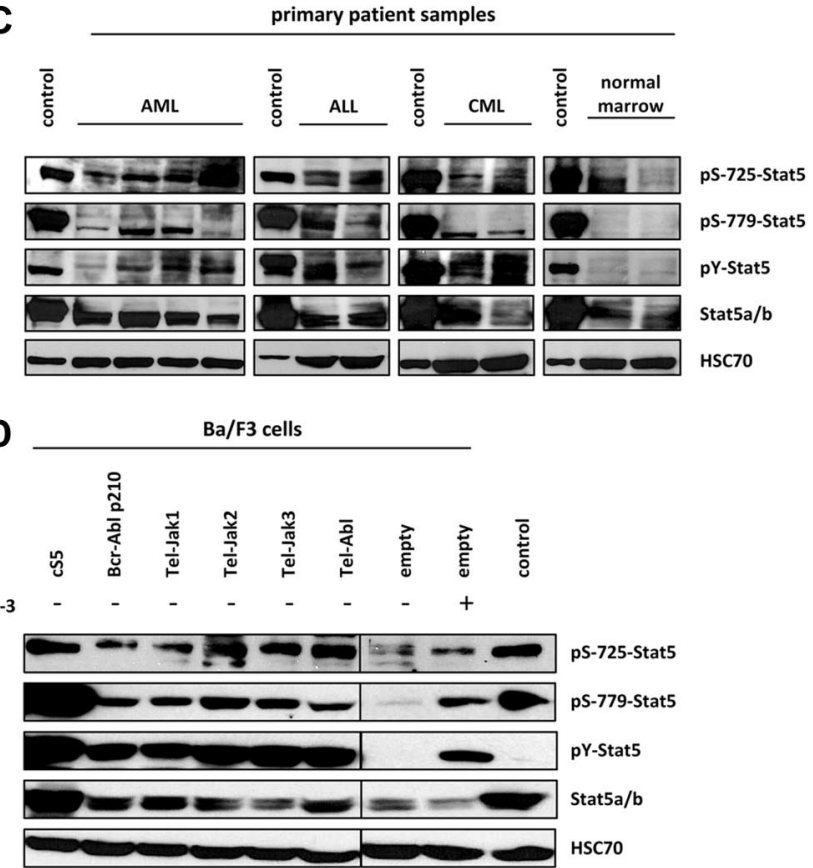

Figure 1. Ser725/779 residues are significantly phosphorylated in transformed cell lines and primary samples from leukemia patients. (A) The C-terminal serine residues 725 and 779 of Stat5a were found to be considerably phosphorylated in human leukemic cell lines. K562 and Ku-812 were used as representative CML samples, and Mv4.11 and MOLM13 for AML. In Western blot analysis, Ser779 was found to be phosphorylated predominantly in CML samples, whereas Ser725 phosphorylation (Abcam antibody) was detected in all leukemia cell lines. S5-expressing gpE +86 fibroblasts served as controls. A representative blot of 3 independent experiments was chosen. (B) Different transformed human T- and B-cell lines, Mac-2A (CTCL), MOLT-4, CCRF-CEM, Jurkat, HPB-ALL (all T-ALL), as well as DAUDI and RAMOS cells (Burkitt-lymphoma), REH (B-ALL), and RL-7 (non-Burkitt lymphoma) cell lines were analyzed for serine phosphorylation by Western blot analysis. Daudi cells as well as CCRF-CEM and Mac-2A cells were found to be strongly positive for Ser725 (Eurogentech and Abcam) and Ser779 phosphorylation. Whereas Mac-2A, Daudi, and to a lesser extent Ramos and REH cells showed Ser779 phosphorylation in addition to Tyr-phosphorylation, Ser779 phosphorylation was largely independent of Tyr-phosphorylation in CCRF-CEM and RL-7 cells. In all B-cell lines except Daudi cells, Ser725 phosphorylation was very low but clearly detectable. A murine T-cell lymphoma line (EL-4) was included in the analysis; it was positive for Ser779 phosphorylation, which was independent of Tyr-phosphorylation. The CML line K562 was used as positive control. Similar results were obtained in 3 individual experiments. (C) Protein analysis of primary samples from AML $(n=4), A L L(n=2)$, and CML $(n=2)$ patients as well as from healthy individuals $(n=2)$ revealed that Stat5a and Stat5b are strongly phosphorylated not only at the critical tyrosine 694 residue, but also at serine 725 , and Stat5a additionally at serine 779 . Serine 779 phosphorylation was not detected in healthy individuals, whereas serine 725 was found to be phosphorylated in all samples tested. GpE+86 cells expressing S5 were used as controls for all analyses. The experiments were performed in duplicate. (D) Ba/F3 cells were engineered by retroviral transduction to overexpress the cS5 variant as well as fusion kinases, which are known to activate Stat5a and Stat5b in human patients. Modified Ba/F3 cells were subjected to immunoblot analysis to evaluate Stat5 tyrosine and serine phosphorylation. Ser725 (Abcam antibody) and Ser779 were strongly phosphorylated in all factor-independent lines. Parental Ba/F3 cells starved for 20 hours and cells restimulated with IL-3, as well as S5-expressing gpE +86 fibroblasts, served as controls (exposed on the same blot; interjacent bands were cut). The blots are representatives of 4 individual experiments.

appeared to enhance tetramer formation, whereas cS5-SASA, similar to WT Stat5a, displayed reduced tetramerization, which might be attributed to diminished oligomer stability. These experiments confirm intact biochemical activity for all constructs, which prompted us to perform cell-culture experiments to test their biologic activity.

\section{Stat5a serine phosphorylation is not essential for mast cell and T-cell proliferation}

We investigated whether the above-mentioned Stat5a mutants were also capable of reconstituting proliferation of primary Stat5- deficient cells, namely Stat5ab ${ }^{\text {null/null }}$ mast cells and $\mathrm{Stat}^{\Delta \mathrm{N} / \Delta \mathrm{N}}$ T cells (Figure 3). For this purpose, mast cells derived from Stat5ab ${ }^{\text {null/null }}$ fetal livers were transduced with different Stat5a retroviral constructs (Figure 3A). As reported by Shelburne et al, ${ }^{36}$ Stat5-deficient mast cells are incapable of proliferating in vitro. Retroviral transduction with WT Stat5a and S5-SASA, however, was found to restore proliferation and compensate for Stat5 deficiency. This was also true for splenic $\mathrm{T}$ cells isolated from Stat $5^{\Delta \mathrm{N} / \Delta \mathrm{N}}$ mice, which fail to proliferate in vitro in response to IL-2 and T-cell receptor activation ${ }^{31,32}$ (Figure 3B). However, re-expression of biologically functional Stat5a serine mutants was 

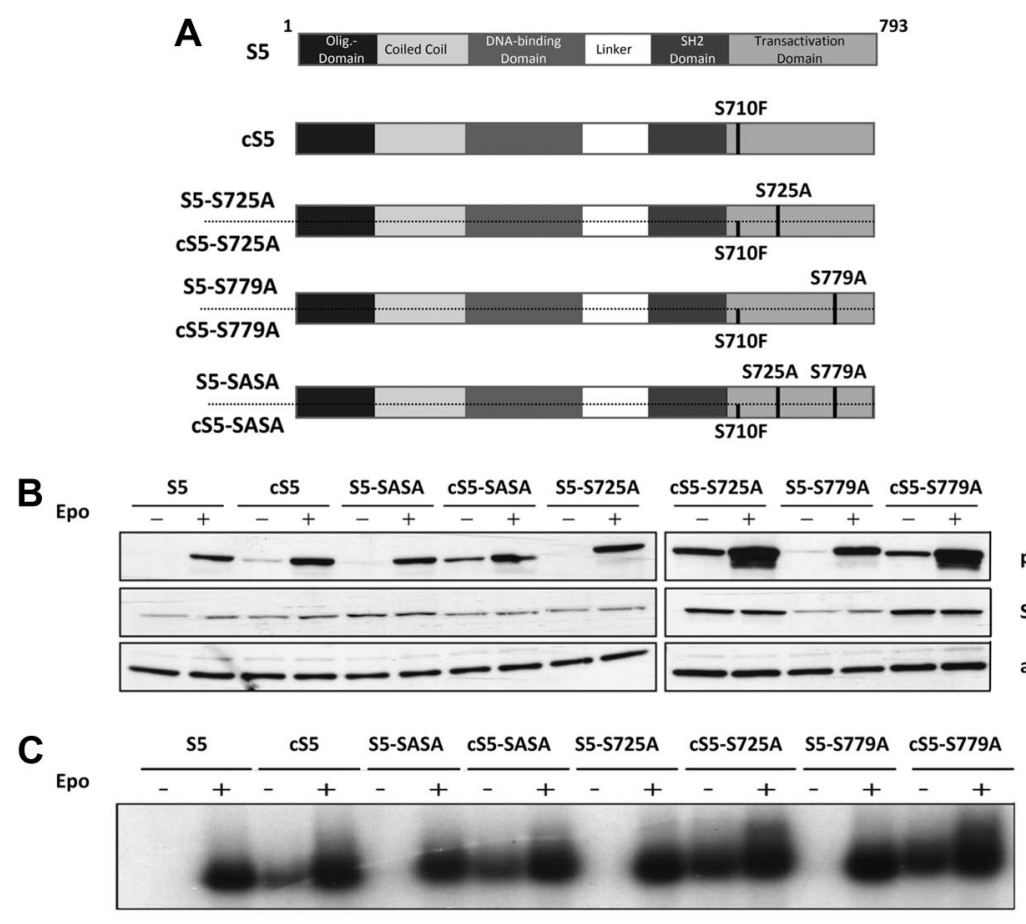

$\beta$-casein

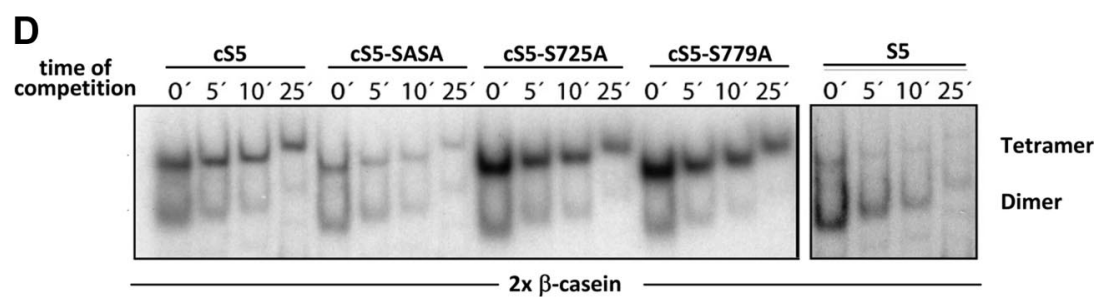

Figure 2. Stat5a mutants exhibit unchanged biochemical properties. (A) Schematic representation of mutant Stat5a proteins. The oncogenic Stat5a mutant cS5 contains a Ser to Phe mutation at position 710 , which renders it constitutively active. The Stat5a mutants S725A, S779A, and SASA carry single or double Ser to Ala substitutions. (B) 293T cells were transfected with the WT (S5) and hyperactive (cS5) mutan Stat5a constructs indicated and stimulated via the erythropoietin (Epo) receptor. Cells were treated $(+)$ with $50 \mathrm{U}$ of Epo or left untreated $(-)$. Whole-cell extracts were prepared and subjected to Western blot analysis to evaluate tyrosine phosphorylation. (C) Epo-stimulated extracts from panel $\mathrm{B}$ were subjected to EMSA using the $\beta$-casein site to study the DNA binding properties of the different WT Stat5a and hyperactive Stat5a mutants. Equal dimer activity was established before analyzing the extracts in tetramer assays as described previously. ${ }^{2,28}$ (D) Tetramer formation of the Stat5a mutants indicated was analyzed with Epo-stimulated 293T pY-Stat5 cell extracts from panel B using a $2 \times \beta$-casein binding element. All Stat5a derivatives were found to bind to the site Stat5a/b as tetramers. Compared with dimers, tetramers were more stable toward competition by cold DNA. The absence of cold DNA (0) represents the saturated binding reaction. This was followed by a $100 \times$ competition by cold DNA for 5,10 , and 25 minutes. cS5, cS5-S725A, and cS5-S779A form tetramers with enhanced DNA binding activity, which was in contrast to WT Stat5a (S5) and cS5-SASA. The data are representative for 3 individual transfections. able to restore T-cell proliferation in culture at levels that were similar for all mutants (Figure 3C). It is of note that in the absence of IL-2, T cells reconstituted with the 4 cS5 mutants showed a higher proliferation rate than cells transduced with WT Stat5a constructs. These findings demonstrate that a lack of serine phosphorylation in Stat5a proteins does not affect their ability to restore Stat5-induced proliferation.

\section{Stat5a serine phosphorylation plays a minor role in lineage repopulation}

To further investigate the role of Stat5a serine phosphorylation in hematopoiesis, an in vivo reconstitution assay was performed. Stat5ab ${ }^{\text {null/null }}$ fetal liver cells (CD45.2) were transduced either with the vector control, with S5, or S5-SASA and transplanted into lethally irradiated CD45.1 recipient mice (Figure 3D-G and supplemental Figure 2A-D). Fetal livers of GFP-transgenic animals with intact Stat5 loci were used as controls (supplemental Figure 2A-D). Analysis of PB and donor chimerism (CD45.1/CD45.2 system $^{30}$ ) performed 8 weeks after transplantation did not reveal significant differences between WT Stat5a and the S5 doubleserine mutant (Figure 3D-G). As in GFP't controls, more than $80 \%$ of $\mathrm{PB}$ cells were donor-derived in the 2 transplant groups (supplemental Figure 2). Moreover, animals reconstituted with S5- and S5-SASA-infected fetal liver cells showed comparable numbers of transduced $\left(\mathrm{GFP}^{+}\right)$donor-derived $\left(\mathrm{CD} 45.2^{+}\right) \mathrm{B}$ cells, T cells, granulocytes, and erythrocytes. Compared with the vector control (supplemental Figure 2), Stat5 deficiency was best compensated for in B-cell and erythroid lineages. The 2 Stat5a variants also compensated for Stat5 deficiency in $\mathrm{CD}^{+}$cells, though to a lesser extent that might be attributable to the lack of Stat5b. In the granulocyte lineage, reconstitution with Stat5a did not have significant beneficial effects. These results indicate that, relative to vector controls, both Stat5a proteins are functional with respect to lineage repopulation.

\section{Constitutively active Stat5a confers factor-independent survival of $\mathrm{Ba} / \mathrm{F} 3$ cells only in the presence of Ser725/779 moieties}

When we analyzed selected aspects of normal Stat5 function, Stat5a serine phosphorylation was not found to be of crucial importance. Therefore, we decided to investigate whether serine phosphorylation is required for the transforming capacity of persistently tyrosine-phosphorylated Stat5a, particularly in view of our findings in human leukemic cell lines. Constitutive activation of Stat5 is known to provide survival and proliferation stimuli that result in cytokine-independent cell expansion. ${ }^{1}$ Hence, all cS5 mutants and the S5 construct were expressed in factor-dependent $\mathrm{Ba} / \mathrm{F} 3$ cells to assess their ability to confer factor-independent proliferation (Figure 4A). Upon cytokine withdrawal, only cells harboring the constitutively active Stat5a mutant with intact serine phosphorylation sites were able to survive (Figure 4B). All other cS5 derivatives underwent apoptosis (Figure 4C), despite the fact that they displayed constitutive phosphorylation of Y694 in the absence of IL-3 (Figure 4D). Thus, cS5 serine mutants exhibit a 
Figure 3. WT and constitutively active Stat5a mutants show full biologic activity in Stat5ab-deficient cells and in a fetalliver cell transplant. (A) Mast cells derived from Stat5abnull/null fetal livers were transduced with Stat5a-expressing retroviral vectors. After transduction, the percentage of $\mathrm{GFP}^{+}$cells was assessed at various points of time. The results obtained on days 3,5 , and 8 and those of days 10,14 , and 16 are summarized in "week 1" and "week 2," respectively. Uninfected cells were used as negative controls without retroviral vector. (B) Unlike primary WT splenocytes, primary Stat5 $5^{\Delta \mathrm{N} / \Delta \mathrm{N}} \mathrm{T}$ cells were not able to proliferate in response to saturating doses of IL-2 and T-cell receptor activation by $\alpha$-CD3. ${ }^{31}$ The values given are representative of 6 independent measurements \pm SEM. (C) Thymidine incorporation assay of starved and IL-2-restimulated T cells. Primary Stat5-deficient T cells (Stat5 ${ }^{\Delta \mathrm{N} / \Delta \mathrm{N}}$ ) were transduced with different retroviral Stat5a constructs labeled with a GFP marker. When Stat $5^{\Delta \mathrm{N} / \Delta \mathrm{N}}$ T cells were reconstituted with WT Stat5a or a biologically functional Stat5a mutant, they fully regained their proliferation capacity, while noninfected cells underwent apoptosis. All WT and constitutively active Stat5a mutants were able to rescue the proliferation defect of Stat5 ${ }^{\Delta \mathrm{N} / \Delta \mathrm{N}} \mathrm{T}$ cells. In contrast to WT mutants, cS5 mutants showed slightly increased thymidine incorporation even without cytokine stimulation. The results represent 4 independent experiments. (D-G) Stat5abnull/null fetal livers (CD45.2) were retrovirally transduced with WT Stat5a (S5) and S5-SASA vectors and transplanted into lethally irradiated CD45.1 recipients. Mice were bled 8 weeks after transplantation and analyzed by FACS for donor engraftment (CD45.2), ${ }^{30}$ GFP expression, and lineage repopulation. Repopulating capacity was assessed for (D) B cells (B220) as well as for (E) T cells (CD8), (F) granulocytes (Gr-1), and (G) erythrocytes (Ter119). For each lineage, marker expression was plotted against GFP expression (left), GFP expression against CD45.2 expression of the donor (middle) and marker against CD45.2 expression (right). Both S5and S5-SASA-reconstituted animals exhibited normal hematopoiesis in all lineages analyzed ( $n=2$ per group).
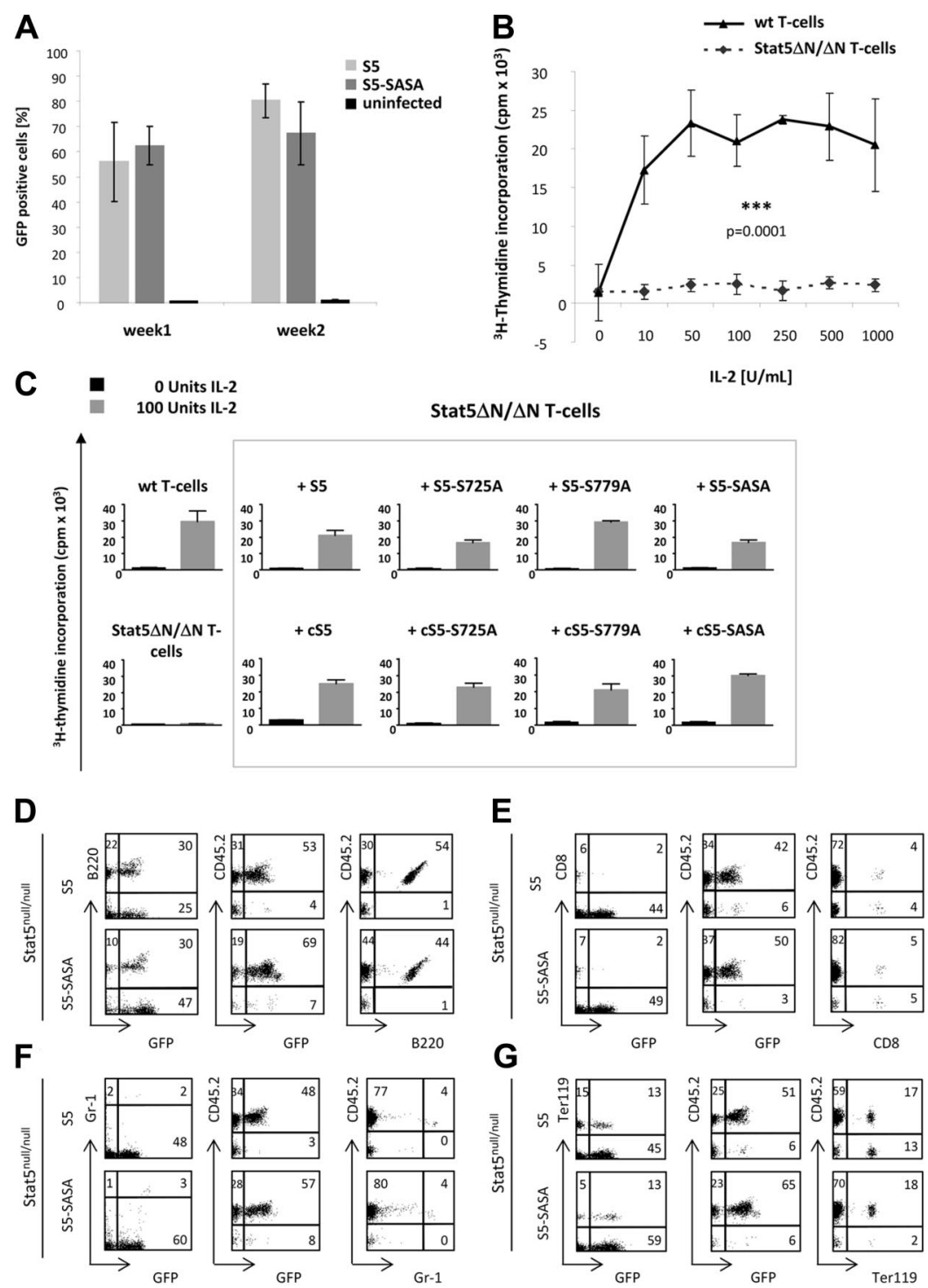

behavior similar to that of parental $\mathrm{Ba} / \mathrm{F} 3$ and S5-transduced cells (Figure 4B-C). These data suggest that, in addition to the constitutive activity of the cS5 mutant, phosphorylation of both serine residues is required to abolish IL-3 dependence of $\mathrm{Ba} / \mathrm{F} 3$ cells.

\section{Loss of serine phosphorylation delays disease onset in transplanted mice}

Because loss of serine phosphorylation proved to have a strong impact on the transforming capacity of oncogenic Stat5a in $\mathrm{Ba} / \mathrm{F} 3$ cells, we investigated its role in a multilineage leukemia transplant model. All constitutively active Stat5a mutants were retrovirally transduced into WT BM cells, and an equal number of transduced LSK-cells (supplemental Table 2) were transplanted into lethally irradiated mice. Flow cytometric analyses of transduced BM cells revealed no lineage skewing due to ectopic expression of any of the retroviral constructs (supplemental Table 2). Strikingly, periodic analysis of PB over 40 weeks demonstrated that mice transplanted with cS5 serine mutants remained disease-free for significantly longer periods than those animals that had received cS5-infected BM cells (Figure 5A). Whereas cS5-transplanted mice succumbed to leukemia within 10 weeks after BM transplantation, single serine mutants developed signs of disease only 4 months after transplantation. Notably, cS5-SASA-transplanted mice remained disease-free throughout the period of analysis (40 weeks), even though $\mathrm{GFP}^{+}$ cells were detected in the PB of all transplant groups.

Multilineage leukemia in cS5-transplanted mice ${ }^{28}$ was marked by a tremendous increase in WBCs, ranging from 15000 to 120000 cells/mL at 8 weeks after transplantation (Figure 5B left panel). FACS analysis demonstrated that this increase was due to myelodysplasia, which was first observed at 4 weeks after transplantation. A dramatic rise in the number of $\mathrm{GFP}^{+}$myeloid cells was detected not only in PB but also in the BM and spleen (Figure 5C and supplemental Figure 3A). Histologically, myelodysplasia in cS5-transplanted mice was confirmed by blood smears and sections of the liver, spleen, and $\mathrm{BM}$ at 8 weeks after transplantation ${ }^{28}$ (Figure 6A).

By contrast, no signs of disease were detectable at 8 weeks after transplantation in animals that had received BM infected with cS5 serine mutants. As measured in the $\mathrm{PB}$, their WBC counts were in the range of those seen in mice transplanted with the vector control (Figure 5B left panel). In flow cytometric analysis, all serine mutants showed a significant number of $\mathrm{GFP}^{+}$cells, most of which 


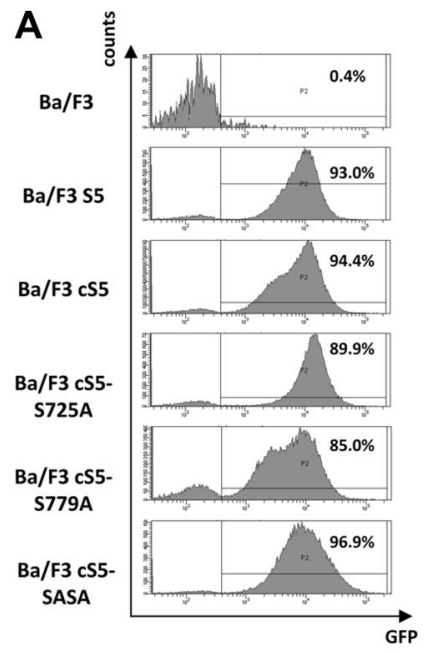

B

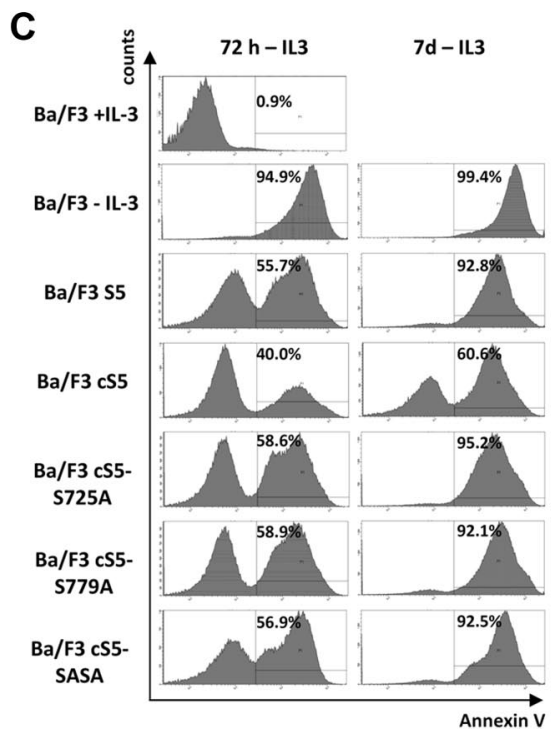

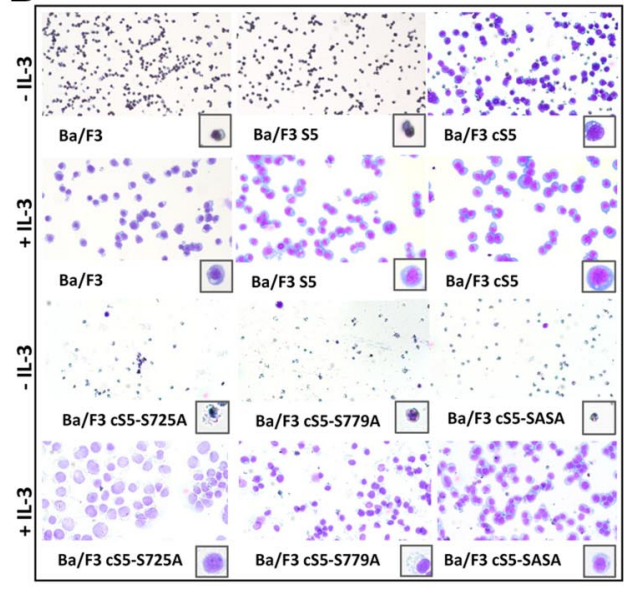

Figure 4. cS5 confers factor-independent survival in the presence of intact serine phosphorylation only. (A) Transduced and FACS-sorted Ba/F3 cells were analyzed for GFP expression by flow cytometry and showed a consistently high percentage of $\mathrm{GFP}^{+}$cells. (B) Representative cytospins of IL-3-dependent $\mathrm{Ba} / \mathrm{F} 3$ cells transduced with the indicated Stat5a variants and kept in medium with/without addition of IL-3. After 7 days, all transduced cells proliferated in the presence of cytokine. However, only cS5-transduced $\mathrm{Ba} / \mathrm{F} 3$ cells were repeatedly able to grow independent of IL-3. Cells transduced with WT Stat5a, cS5-S725A, cS5-S779A, and cS5-SASA were not transformed to factor independence and underwent apoptosis similar to untransduced Ba/F3 controls without IL-3. (C) Growth factordependent $\mathrm{Ba} / \mathrm{F} 3$ cells were transduced with the different cS5 constructs and WT Stat5a as indicated and FACS-sorted for $\mathrm{GFP}^{+}$cells. Upon IL-3 withdrawal, apoptosis was analyzed by FACS using an annexin $V$ antibody at 72 hours and at 7 days. cS5-expressing cells were the only ones able to survive without cytokine stimulation. All other mutants underwent apoptosis after factor depletion in 3 individual experiments. (D) Cell lysates from sorted $\mathrm{GFP}^{+}$cells were prepared and analyzed by immunoblotting with the indicated antibodies. Parental Ba/F3 cells showed weak expression of total Stat5, which was strongly tyrosine phosphorylated upon IL-3 stimulation. In all Ba/F3 cells transduced with the different cS5 constructs, Stat5 was highly tyrosine phosphorylated in the absence of cytokine stimulation.

were $\mathrm{CD} 3^{+}$lymphoid cells and $\mathrm{Gr}-1^{+}$myeloid cells (Figure $5 \mathrm{C}$ left panel). At 8 weeks after transplantation, histologic analysis of mice injected with serine mutants yielded similar results as for vector controls (Figure 6A). Even at 32 weeks after transplantation, the WBC counts of single-serine mutants were only slightly elevated compared with vector controls (Figure 5B right panel). However, the overall percentage of $\mathrm{GFP}^{+}$cells in Stat5a single-serine mutants increased to $30 \%$ to $40 \%$ of WBCs. Both in the PB and in the spleen, this increase was more pronounced in the $\mathrm{CD}^{+}$and $\mathrm{Gr}-1^{+}$population than in the $\mathrm{Gr}-1^{+} \mathrm{Mac}-1^{+}$myeloid cells (Figure $5 \mathrm{C}$ right panel and supplemental Figure $3 \mathrm{~A}$ ). The absence of $\mathrm{GFP}^{+}$ Gr- $1^{+}$Mac- $1^{+}$myeloid cells in PB cannot be explained by an altered total number of myeloid cells at any point of analysis, because it was not different from that observed in vector controls (supplemental Table 3). Interestingly, the total number of lymphoid cells in the PB of single-serine mutants was not affected by the increase in $\mathrm{GFP}^{+}$cells either. Conversely, the increase in $\mathrm{GFP}^{+}$ cells in terminally ill cS5-transplanted mice resulted in a higher total number of myeloid cells. In the BM of both single-serine transplant groups the number of $\mathrm{GFP}^{+}$cells was too low to be assigned to a specific lineage (supplemental Figure 3A). This was also true for all samples obtained from cS5-SASA-transplanted mice (Figure 5C right panel and supplemental Figure 3A). No increase in the $\mathrm{GFP}^{+}$population was seen in vector controls
(Figure 5C right panel; data not shown for BM and spleen). At 32 weeks, an increased number of lymphoid cells was also detected in the blood smears of cS5 single-serine mutants (Figure 6B). Comparing the 2 mutants, cS5-S725A-transplanted mice were found to have a slightly stronger disease phenotype with additional disruption of spleen architecture and infiltration of the liver by hematopoietic cells. cS5-SASA-transplanted mice did not show any signs of disease throughout the period of analysis (40 weeks). In Western blot analyses, significant Stat5a Ser779 phosphorylation was detected in the spleens of diseased cS5 and cS5-S725A mice, whereas there was no significant Stat5 serine phosphorylation in cS5-S779A-transplanted mice and disease-free animals (supplemental Figure 3B). It should be noted that the difference in Ser779 phosphorylation levels of the $2 \mathrm{cS} 5$ mice was due to their different disease stages. Because the lack of C-terminal serine phosphorylation was found to prolong disease-free survival in mice, these results strongly suggest a role of Stat5 serine phosphorylation in myeloid dysplasia.

\section{Discussion}

Constitutive activation of Stat5 was shown to be directly involved in oncogenic transformation. ${ }^{1-3}$ In addition to activating tyrosine 
Figure 5. Loss of Stat5a serine-phosphorylation delays disease onset in transplanted mice. (A) Kaplan-Meier plot of cS5- vs cS5-S725A-, cS5-S779A-, and cS5-SASAtransplanted mice. GFP vector-transplanted mice were included as controls. All cS5-transplanted mice died within 10 weeks. In contrast, cS5-SASA-transplanted mice remained disease-free, and disease onset in cS5 single serine mutants was significantly delayed $\left({ }^{\star * \star} P<.001\right)$. All groups were monitored over a period of 40 weeks after which all mice were killed and analyzed $(n \geq 8)$. (B) WBC counts of transplanted mice at 8 weeks (left) and 32 weeks after transplantation (right). PB hematology revealed a dramatic increase in WBCs in cS5-transplanted mice after 8 weeks. By contrast, all serine mutants showed a slight increase in WBCs after 32 weeks only. (C) FACS analysis of PB for representative cS5 versus CS5-S725A-, cS5-S779A-, and cS5-SASA-transplanted mice at 8 and 32 weeks after BM transplantation. The overall number of $\mathrm{GFP}^{+}$cells is displayed in the histogram. All cS5-transplanted mice were found to present with severe myelodysplasia when $\mathrm{GFP}^{+}$ cells were gated for lymphoid (CD3 and CD19) and myeloid (Gr-1 and Mac-1/CD11b) markers, respectively. Conversely, all serine mutants showed an increase in $\mathrm{GFP}^{+}$lymphoid cells (mainly $\mathrm{CD}^{+}$cells) and in $\mathrm{Gr}-1^{+}$cells.

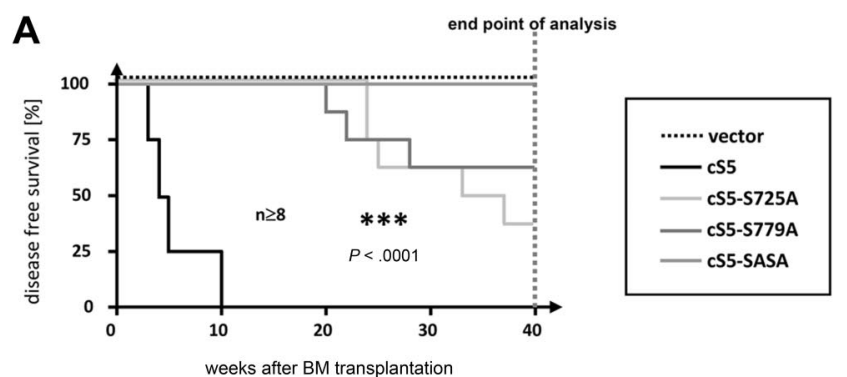

B 8 weeks after transplantation

32 weeks after transplantation
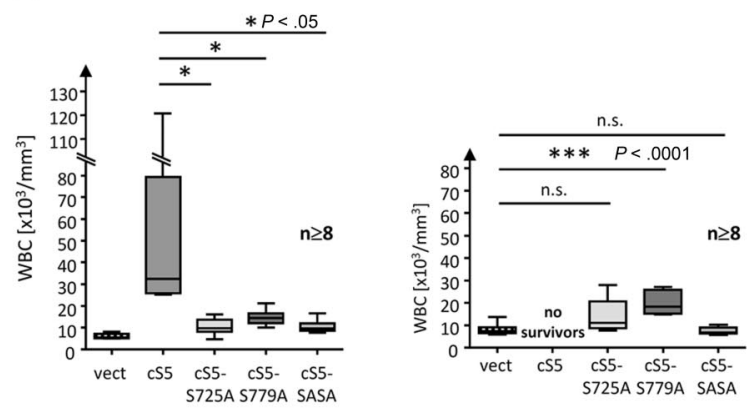

C

8 weeks after transplantation
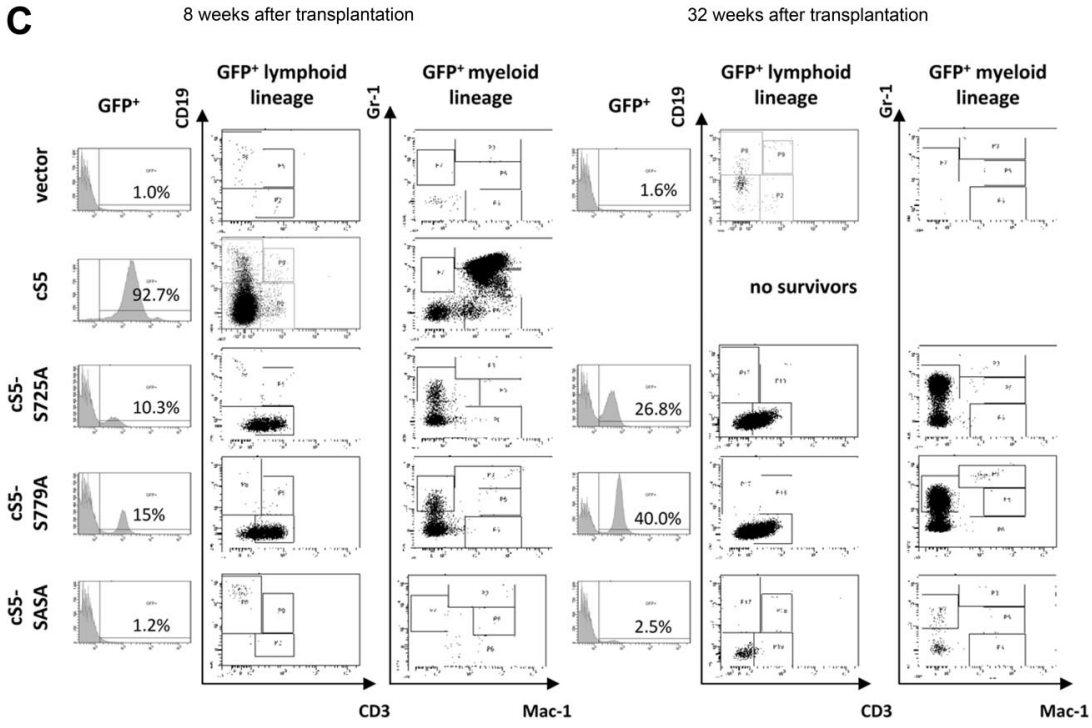

phosphorylation, serine phosphorylation has been found in most Stat proteins. The latter has been shown to be important for maximal transcriptional activity of Stat1/3/4 but not of Stat5a and Stat5b. ${ }^{22}$ Biochemical and biologic functions of Stat serine phosphorylation also include DNA binding, ${ }^{23,37}$ cofactor association, ${ }^{38}$ immune responses, ${ }^{39,40}$ and transformation. ${ }^{41,42}$ Interestingly, we found the C-terminally mapped serines of Stat5a (Ser725/779) to be highly phosphorylated in human bcr/abl ${ }^{+} \mathrm{CML}$ samples, human lymphoma cell lines, primary leukemia patient samples, and $\mathrm{Ba} / \mathrm{F} 3$ cells overexpressing different fusion tyrosine kinases. It is of note that strong Ser779 phosphorylation was detected in primary AML, ALL, and CML patient samples, but not in healthy individuals. These findings suggest that Stat5a serine phosphorylation may have an essential role in hematopoietic transformation.

To evaluate the importance of Stat5a serine phosphorylation at residues 779 and 725 for hematopoietic cells, different WT (S5) and point-mutated hyperactive Stat5a (cS5) variants exhibiting intact biochemical activity were used. Notably, all hyperactivated mutants showed the same capacity to form tetramers, which is essential for the leukemogenic potential of Stat5. ${ }^{28}$ Similar to serine mutants of human Stat5a, ${ }^{43}$ all WT and hyperactive constructs were able to rescue the phenotype of primary Stat5-deficient splenocytes. Likewise, reconstitution of lethally irradiated mice with Stat5-deficient fetal liver cells harboring the double-serine mutant of WT Stat5a resulted in lineage repopulation comparable with that seen in mice transplanted with Stat5a-transduced fetal liver cells. As in Stat5ab ${ }^{-/-}$animals, ${ }^{17,44-46}$ transplantation of Stat5 $5^{\text {null/null }}$ fetal livers induced lymphopenia and anemia. Unlike vector controls, both Stat5a variants were able to compensate for Stat 5 deficiency in $\mathrm{B}$, T, and erythroid cell lineages. These data suggest that serine phosphorylation has only a minor role in normal hematopoiesis, which is in line with previous reports. ${ }^{11,23-25}$ Yet, the significance of serine phosphorylation for other aspects of hematopoiesis, such as homing, migration, and stress hematopoiesis still remains to be elucidated.

Intriguingly, the present study revealed that phosphorylation of both serines is a prerequisite for factor-independent growth of $\mathrm{Ba} / \mathrm{F} 3$ cells. Mutation of either of the 2 serine residues was found to 
A 8 weeks after transplantation

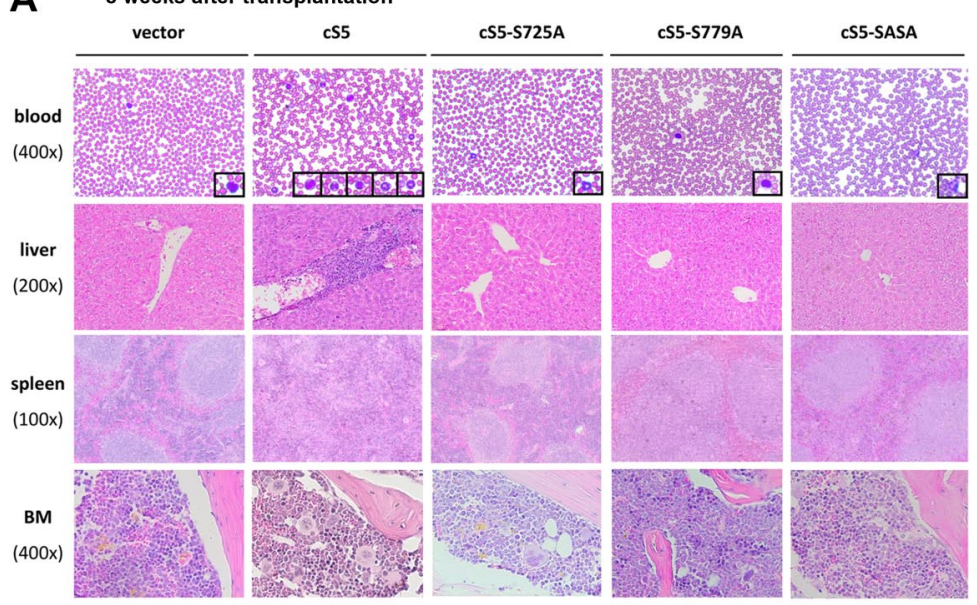

B 32 weeks after transplantation

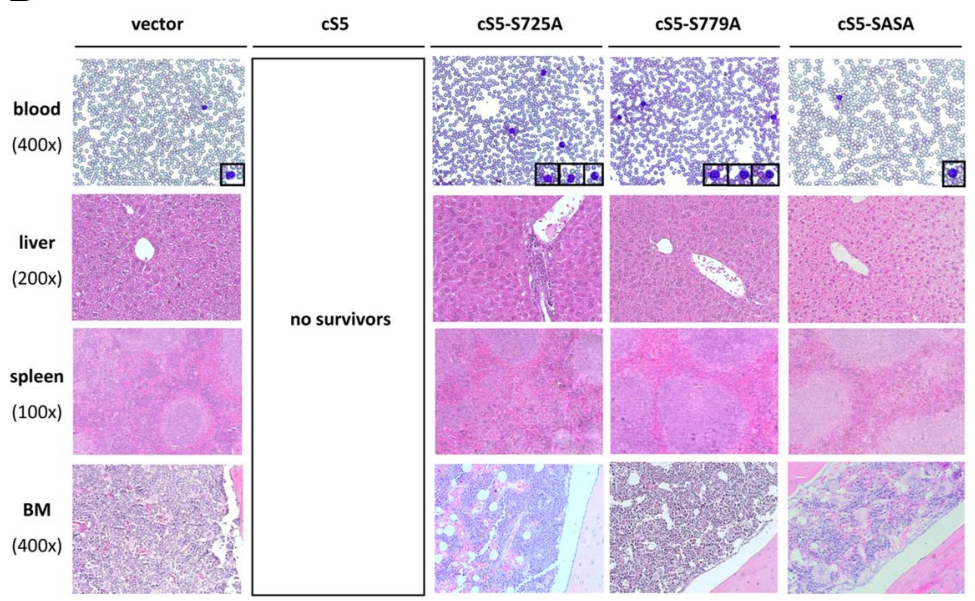

Figure 6. Alterations of organotypic structures in cS5-transplanted mice and cS5 serine mutants. (A) At 8 weeks after BM transplantation, histopathologic analysis of PB, liver, spleen, and BM revealed signs of leukemia in CS5-transplanted mice, including elevated numbers of myeloid and lymphoid cells in the PB and disruption of spleen germinal center architecture with concomitant splenomegaly. In addition, the liver portal tracts and parts of the liver parenchyma in these mice were diffusely infiltrated by hematopoietic cells. Blast-like cells were detected in the BM. Contrary, mice transplanted with cS5 serine mutants did not show any abnormalities and histologically resembled the GFP vector controls. (B) At 32 weeks after transplantation, mice transplanted with cS5 single-serine mutants showed increased numbers of lymphoid cells in PB, with cS5S725A-transplanted mice displaying a stronger phenotype characterized by disruption of the splenic architecture and infiltration of the liver by hematopoietic cells. cS5-SASA-transplanted mice did not show any signs of disease throughout the period of analysis. For each transplant group, a minimum of 8 mice was analyzed, which all displayed similar histologic features (magnifications: spleen $\times 100$, liver $\times 200$, BM and blood smear $\times 400)$. abolish factor-independent survival of $\mathrm{Ba} / \mathrm{F} 3$ cells, even though Stat5 was highly tyrosine phosphorylated. This suggests that Stat5a serine phosphorylation is essential for the transformation of hematopoietic cells. Indeed, loss of Stat5a serine phosphorylation had a strong impact on the transforming capacity of constitutively active Stat5a in a BM transplant model. Substitution of a single C-terminal serine by alanine proved sufficient to significantly delay disease onset in cS5-transplanted mice, whereas mutation of both $\mathrm{C}$-terminal serines abrogated leukemia development. Integration of all retroviral cS5-constructs including cS5-SASA was verified in splenocytes of transplanted mice (by sequencing retroviral insertion; data not shown), and $\mathrm{GFP}^{+}$cells were traceable in all animals throughout the period of analysis. Interestingly, we observed a lineage shift in mice transplanted with serine-mutant cS5 variants. The serine mutants did not show expansion of the myeloid lineage, as was observed in cS5-transplanted animals, but an increase in $\mathrm{CD}^{+}$population. This was not due to a reduction in the total number of myeloid cells, which was similar to that found in vector controls. Presumably, it was the shift of GFP expression to the lymphoid lineage that prevented myeloid expansion and thus rapid onset of disease. Skewing to specific lineages and differences in transduction rates during the initial culturing and infection phase were precluded by administering the same number of transduced LSK cells to each animal.

The reduced transforming capacity of cS5 serine mutants might be explained by alterations in the transcriptional profile. The serine phosphorylation sites are in close vicinity to the amphipathic $\alpha$-helix within the transactivation domain of Stat5a, which is negatively charged. ${ }^{34}$ Stat5a and Stat5b contain only weak transactivation domains. ${ }^{34,47}$ This is why Stat5-mediated transcription requires binding of factors that coregulate gene expression cell specifically. ${ }^{34}$ Serine phosphorylation at the C-terminal tail of Stat5 proteins might be essential for creating a more acidic, negatively charged surface that is able to attract positively charged cofactors or other transcription factors. Alternatively, unknown factors such as different cellular location of Stat $5 \mathrm{a}^{41,48}$ or altered accessibility for tyrosine phosphatases might contribute to the reduced transforming capacity of serine mutants.

In conclusion, our data suggest that Stat5a Ser725/779 phosphorylation is indispensible for hematopoietic transformation. As it is not conserved in Stat5b, the region of Stat5a encompassing Ser779 might provide a particularly attractive target for future therapeutic strategies in leukemia and myeloid dysplasias. Thus, identification of the kinase responsible for phosphorylating Ser779 in Stat5a would be the first essential step toward the development of a suitable inhibitor. Unlike Stat1 and Stat3, Stat5 proteins are not considered substrates of extracellular signal-regulated kinases (ERKs) that bind to PSP motifs. ${ }^{24,49,50}$ Hence, Stat5 regulation may be different from that of other Stats. ${ }^{51}$ There are several kinases that may control Stat5a serine phosphorylation, including stress kinases, cyclin-dependent kinases (CDKs), and Pim kinases. Elucidating the impact of eliminating serine phosphorylation in transformed cells might, therefore, be a step forward in the development 
of a novel therapeutic concept for hematopoietic malignancies that has no major side effects on normal hematopoiesis.

\section{Acknowledgments}

We thank G. Stengl for her excellent technical support and V. Penard-Lacronique for providing us with the Tel-fusion constructs used in the $\mathrm{Ba} / \mathrm{F} 3$ cell experiments. The authors are indebted to I. and M. Friedbichler (Innsbruck Medical University, Innsbruck, Austria) for providing editorial assistance in the preparation of the manuscript.

This work was funded by grant Sonderforschungsbereich (SFB) F28 from the Austrian Basic Research Funds (FWF) to R.M., K.F., B.K., H.B., A.H., S.F., V.S., E.W.M.; Wiener Wissenschafts-, Forschungs- und Technologiefonds (WWTF) grant LS07-037 to V.S.; Herzfelder Family Foundation and FWF grant WK-001 to M.A.K. and E.W.M.; Austrian Federal Ministry for Education,
Science, and Culture grant GZ 200.062/2-VI/1/2002 to S.C.-R. and P.V.; Association de la Recherche contre le Cancer (ARC) grant to F.G.; and National Institutes of Health (NIH) grant R01DK059380 to K.D.B.

\section{Authorship}

Contribution: K.F., M.A.K., B.K., G.L., A.H., S.Y., S.F., and S.C.-R. performed the experiments; K.F. and R.M. designed the experiments, interpreted the results, and wrote the paper; H.B., V.S., K.D.B., F.G., P.V., and E.W.M. contributed essential reagents; and V.S., M.A.K., K.D.B., and F.G. revised the manuscript.

Conflict-of-interest disclosure: The authors declare no competing financial interests.

Correspondence: Richard Moriggl, Ludwig Boltzmann Institute for Cancer Research (LBI-CR), Waehringer Str 13a, A-1090 Vienna, Austria; e-mail: richard.moriggl@lbicr.lbg.ac.at.

\section{References}

1. Bunting KD. STAT5 signaling in normal and pathologic hematopoiesis. Front Biosci. 2007;12: 2807-2820.

2. Kornfeld JW, Grebien F, Kerenyi MA, et al. The different functions of Stat5 and chromatin alteration through Stat5 proteins. Front Biosci. 2008; 13:6237-6254.

3. Hennighausen L, Robinson GW. Interpretation of cytokine signaling through the transcription factors STAT5A and STAT5B. Genes Dev. 2008; 22(6):711-721.

4. Nosaka T, Kitamura T. Janus kinases (JAKs) and signal transducers and activators of transcription (STATs) in hematopoietic cells. Int J Hematol. 2000;71(4):309-319.

5. Grimley PM, Dong F, Rui H. Stat5a and Stat5b: fraternal twins of signal transduction and transcriptional activation. Cytokine Growth Factor Rev. 1999;10(2):131-157.

6. Coffer PJ, Koenderman L, de Groot RP. The role of STATs in myeloid differentiation and leukemia Oncogene. 2000;19(21):2511-2522.

7. Schuringa JJ, Wu K, Morrone G, Moore MA. Enforced activation of STAT5A facilitates the generation of embryonic stem-derived hematopoietic stem cells that contribute to hematopoiesis in vivo. Stem Cells. 2004;22(7):1191-1204.

8. Gatzka M, Piekorz R, Moriggl R, Rawlings J, Ihle JN. A role for STAT5A/B in protection of peripheral T-lymphocytes from postactivation apoptosis: insights from gene expression profiling. Cytokine. 2006;34(3-4):143-154.

9. Zeng R, Aoki Y, Yoshida M, Arai K, Watanabe S. Stat5B shuttles between cytoplasm and nucleus in a cytokine-dependent and -independent manner. J Immunol. 2002;168(9):4567-4575.

10. Iyer J, Reich NC. Constitutive nuclear import of latent and activated STAT5a by its coiled coil domain. FASEB J. 2008;22(2):391-400.

11. Yamashita $\mathrm{H}, \mathrm{Xu}$ J, Erwin RA, Farrar WL, Kirken RA, Rui H. Differential control of the phosphorylation state of proline-juxtaposed serine residues Ser725 of Stat5a and Ser730 of Stat5b in prolactin-sensitive cells. J Biol Chem. 1998; 273(46):30218-30224.

12. Van Etten RA. Aberrant cytokine signaling in leukemia. Oncogene. 2007;26(47):6738-6749.

13. Levine RL, Pardanani A, Tefferi A, Gilliland DG. Role of JAK2 in the pathogenesis and therapy of myeloproliferative disorders. Nat Rev Cancer. 2007;7(9):673-683.

14. Morgan KJ, Gilliland DG. A role for JAK2 mutations in myeloproliferative diseases. Annu Rev Med. 2008;59:213-222.
15. Carlesso N, Frank DA, Griffin JD. Tyrosyl phosphorylation and DNA binding activity of signal transducers and activators of transcription (STAT) proteins in hematopoietic cell lines transformed by Bcr/Abl. J Exp Med. 1996;183(3):811-820.

16. Ilaria RL Jr, Van Etten RA. P210 and P190 (BCR/ $A B L$ ) induce the tyrosine phosphorylation and DNA binding activity of multiple specific STAT family members. J Biol Chem. 1996;271(49): 31704-31710.

17. Hoelbl A, Kovacic B, Kerenyi MA, et al. Clarifying the role of Stat5 in lymphoid development and Abelson-induced transformation. Blood. 2006; 107(12):4898-4906.

18. Nyga $\mathrm{R}$, Pecquet $\mathrm{C}$, Harir $\mathrm{N}$, et al. Activated STAT5 proteins induce activation of the PI 3-kinase/Akt and Ras/MAPK pathways via the Gab2 scaffolding adapter. Biochem J. 2005;390(Pt 1): 359-366.

19. Harir $\mathrm{N}$, Pecquet $\mathrm{C}$, Kerenyi $\mathrm{M}$, et al. Constitutive activation of Stat5 promotes its cytoplasmic localization and association with PI3-kinase in myeloid leukemias. Blood. 2007;109(4):1678-1686.

20. Harir N, Boudot C, Friedbichler K, et al. Oncogenic kit controls neoplastic mast cell growth through a Stat5/PI3-kinase signaling cascade. Blood. 2008;112(6):2463-2473.

21. Li G, Miskimen KL, Wang Z, et al. STAT5 requires the N-domain for suppression of miR15/16, induction of bcl-2, and survival signaling in myeloproliferative disease. Blood. 2010;115(7):14161424.

22. Decker T, Kovarik P. Serine phosphorylation of STATs. Oncogene. 2000;19(21):2628-2637.

23. Beuvink I, Hess D, Flotow H, Hofsteenge J, Groner B, Hynes NE. Stat5a serine phosphorylation. Serine 779 is constitutively phosphorylated in the mammary gland, and serine 725 phosphorylation influences prolactin-stimulated in vitro DNA binding activity. J Biol Chem. 2000;275(14): 10247-10255.

24. Kirken RA, Malabarba MG, Xu J, et al. Prolactin stimulates serine/tyrosine phosphorylation and formation of heterocomplexes of multiple Stat5 isoforms in Nb2 lymphocytes. J Biol Chem. 1997; 272(22):14098-14103.

25. Park SH, Yamashita H, Rui H, Waxman DJ. Serine phosphorylation of $\mathrm{GH}$-activated signal transducer and activator of transcription $5 \mathrm{a}$ (STAT5a) and STAT5b: impact on STAT5 transcriptional activity. Mol Endocrinol. 2001;15(12): 2157-2171.

26. Yamashita H, Nevalainen MT, Xu J, et al. Role of serine phosphorylation of Stat5a in prolactin- stimulated beta-casein gene expression. Mol Cell Endocrinol. 2001;183(1-2):151-163.

27. Weaver AM, Silva CM. S731 in the transactivation domain modulates STAT5b activity. Biochem Biophys Res Commun. 2007;362(4):1026-1030.

28. Moriggl R, Sexl V, Kenner L, et al. Stat5 tetramer formation is associated with leukemogenesis. Cancer Cell. 2005;7(1):87-99.

29. Grebien F, Kerenyi MA, Kovacic B, et al. Stat5 activation enables erythropoiesis in the absence of EpoR and Jak2. Blood. 2008;111(9):45114522.

30. Li G, Wang Z, Zhang Y, et al. STAT5 requires the $\mathrm{N}$-domain to maintain hematopoietic stem cell repopulating function and appropriate lymphoidmyeloid lineage output. Exp Hematol. 2007; 35(11):1684-1694.

31. Moriggl R, SexI V, Piekorz R, Topham D, Ihle JN Stat5 activation is uniquely associated with cytokine signaling in peripheral T cells. Immunity. 1999;11(2):225-230.

32. Moriggl R, Topham DJ, Teglund S, et al. Stat5 is required for IL-2-induced cell cycle progression of peripheral T cells. Immunity. 1999;10(2):249-259.

33. Onishi M, Nosaka T, Misawa K, et al. Identification and characterization of a constitutively active STAT5 mutant that promotes cell proliferation. Mol Cell Biol. 1998;18(7):3871-3879.

34. Moriggl R, Gouilleux-Gruart V, Jahne R, et al. Deletion of the carboxyl-terminal transactivation domain of MGF-Stat5 results in sustained DNA binding and a dominant negative phenotype. $\mathrm{Mol}$ Cell Biol. 1996;16(10):5691-5700.

35. Wang D, Moriggl R, Stravopodis D, et al. A smal amphipathic alpha-helical region is required for transcriptional activities and proteasome-dependent turnover of the tyrosine-phosphorylated Stat5. Embo J. 2000;19(3):392-399.

36. Shelburne CP, McCoy ME, Piekorz R, et al. Stat5 expression is critical for mast cell development and survival. Blood. 2003;102(4):1290-1297.

37. Zhang X, Blenis J, Li HC, Schindler C, Chen-Kiang S. Requirement of serine phosphorylation for formation of STAT-promoter complexes. Science. 1995;267(5206):1990-1994.

38. Zakharova N, Lymar ES, Yang E, et al. Distinct transcriptional activation functions of STAT1alpha and STAT $1 \beta$ on DNA and chromatin templates. J Biol Chem. 2003;278(44):43067-43073.

39. Varinou L, Ramsauer K, Karaghiosoff M, et al. Phosphorylation of the Stat1 transactivation domain is required for full-fledged IFN- $\gamma$-dependent innate immunity. Immunity. 2003;19(6):793-802. 
40. Pilz A, Kratky W, Stockinger S, et al. Dendritic cells require STAT-1 phosphorylated at its transactivating domain for the induction of peptidespecific CTL. J Immunol. 2009;183(4):2286-2293.

41. Gough DJ, Corlett A, Schlessinger K, Wegrzyn J, Larner AC, Levy DE. Mitochondrial STAT3 supports Ras-dependent oncogenic transformation. Science. 2009;324(5935):1713-1716.

42. Frank DA, Mahajan S, Ritz J. B lymphocytes from patients with chronic lymphocytic leukemia contain signal transducer and activator of transcription (STAT) 1 and STAT3 constitutively phosphorylated on serine residues. J Clin Invest. 1997; 100(12):3140-3148

43. Xue HH, Fink DW Jr, Zhang X, Qin J, Turck CW, Leonard WJ. Serine phosphorylation of Stat5 proteins in lymphocytes stimulated with IL-2. Int Immunol. 2002;14(11):1263-1271.

44. Yao Z, Cui Y, Watford WT, et al. Stat5a/b are es- sential for normal lymphoid development and differentiation. Proc Natl Acad Sci U S A. 2006; 103(4):1000-1005

45. Socolovsky M, Fallon AE, Wang S, Brugnara C, Lodish HF. Fetal anemia and apoptosis of red cell progenitors in Stat5a-1-5b-/- mice: a direct role for Stat5 in Bcl-X(L) induction. Cell. 1999; 98(2):181-191.

46. Socolovsky M, Nam H, Fleming MD, Haase VH, Brugnara C, Lodish HF. Ineffective erythropoiesis in Stat5a(-/-) $5 \mathrm{~b}(-/-)$ mice due to decreased survival of early erythroblasts. Blood. 2001; 98(12):3261-3273.

47. Moriggl R, Berchtold S, Friedrich K, et al. Comparison of the transactivation domains of Stat5 and Stat6 in lymphoid cells and mammary epithelial cells. Mol Cell Biol. 1997;17(7):3663-3678.

48. Wegrzyn J, Potla R, Chwae YJ, et al. Function of mitochondrial Stat3 in cellular respiration. Science. 2009;323(5915):793-797.

49. Beadling $\mathrm{C}, \mathrm{Ng}$ J, Babbage JW, Cantrell DA. Interleukin-2 activation of STAT5 requires the convergent action of tyrosine kinases and a serine/ threonine kinase pathway distinct from the Raf1/ ERK2 MAP kinase pathway. Embo J. 1996;15(8): 1902-1913.

50. Wartmann M, Cella N, Hofer P, et al. Lactogenic hormone activation of Stat5 and transcription of the beta-casein gene in mammary epithelial cells is independent of p42 ERK2 mitogen-activated protein kinase activity. J Biol Chem. 1996; 271(50):31863-31868.

51. Nagy ZS, Wang Y, Erwin-Cohen RA, et al. Interleukin-2 family cytokines stimulate phosphorylation of the Pro-Ser-Pro motif of Stat5 transcription factors in human T cells: resistance to suppression of multiple serine kinase pathways. J Leukoc Biol. 2002;72(4):819-828. 